\title{
Avaliação do Módulo de Identificação da Polaridade Geral dos Comentários do TripAdvisor ${ }^{\circledR}$
}

\author{
Taylor Santos Oliveira ${ }^{1}$, Dionnys Santos Marinho ${ }^{1}$, Parcilene Fernandes Brito ${ }^{1}$ \\ ${ }^{1}$ Ciências da computação - Centro Universitário Luterano de Palmas CEULP/ULBRA \\ Avenida Teotônio Segurado, 1501 SUL, CEP 77.019-900, Palmas - TO - Brasil \\ \{uaitayloroliveira, dionnys.marinho, parcilene\}@gmail.com
}

\begin{abstract}
Resumo. Este artigo apresenta o processo de avaliação do módulo de identificação da polaridade geral dos comentários de um site de turismo no contexto nacional. $O$ processo realizado foi dividido em quatro etapas. A primeira foi a de análise manual de 70 comentários. A segunda etapa foi a criação de uma matriz de confusão para comparação do resultado encontrado na análise manual e do sistema. A terceira etapa foi realizado a aplicação das métricas "Precision" $e$ "Recall" para a avaliação de desempenho no que concerne à verificação da polaridade do comentário. Por fim, foi feita a análise de correlação entre o resultado obtido pelo sistema e o valor da avaliação do usuário via escala Likert. Para isso, o processo teve como entrada os dados das avaliações do TripAdvisor que estão contidos na base de dados da versão 2 da ferramenta SentimentALL.
\end{abstract}

\section{Introdução}

Este trabalho objetiva apresentar o processo de avaliação do módulo de inferência da polaridade geral dos comentários do TripAdvisor ${ }^{\circledR}$ realizado em Oliveira, Marinho e Brito (2018) a partir dos aspectos polarizados identificados no trabalho de Araújo (2017) . Este módulo é parte do projeto SentimentALL apresentado em sua totalidade em Brito (2018), em que são utilizadas técnicas computacionais de Análise de Sentimentos e abordagens psicológicas de Análise Comportamental aplicadas no contexto do Turismo Nacional. O ambiente estudado neste trabalho é o site especializado em turismo TripAdvisor ${ }^{\circledR}$ (https://www.tripadvisor.com.br/). O TripAdvisor ${ }^{\circledR}$ é um site de viagens que fornece informações e opiniões de conteúdos relacionados ao turismo.

O módulo de inferência da polaridade geral dos comentários de Oliveira, Marinho e Brito (2018) tem como entrada um conjunto de aspectos (por exemplo, atendimento, comida, quarto etc.) avaliados como positivos ou negativos extraídos dos comentários de avaliações de usuários do site TripAdvisor ${ }^{\circledR}$, a partir da ferramenta SentimentALL (ARAÚJO, 2017; BRITO, 2018). O processo de inferência da polaridade geral apresentado em Oliveira, Marinho e Brito (2018) considerou dois pontos: a incidência do aspecto no total geral de comentários (quanto maior a incidência maior a relevância, entendida como um peso adicionado ao aspecto), e os aspectos de cada comentário (com sua devida polaridade). Assim, cada aspecto positivo ou negativo de um comentário multiplica a seu valor (1 ou -1, conforme o caso) o peso do aspecto identificado anteriormente. Com a soma de todos os valores de todos os aspectos de um comentário, identifica-se a polaridade geral do comentário, que pode ser positiva ou negativa. É essa polaridade geral que foi avaliada neste artigo.

Para analisar o desempenho do sistema em relação a identificação da polaridade geral do comentário são utilizadas as métricas Precision, Recall e F-Measure juntamente com uma estratégia de organização de informações conhecida como "Matriz de Confusão", além de verificar a existência da correlação entre o resultado do módulo com a informação da avaliação do usuário via escala Likert. A matriz de confusão consiste em uma tabela de cruzamento de informações que apresenta visualmente o número de acertos (Verdadeiros- 
Positivos ou Vp), erros (Falsos-Positivos ou Fp), perdas (Falsos-Negativos ou Fn) e rejeições corretas (Verdadeiros-Negativos ou VN) (BAKER, 2008). Dessa forma, os termos utilizados para os cálculos são nitidamente identificados, facilitando a aplicação das técnicas de avaliação.

Na seção seguinte são apresentados de forma sucinta conceitos relacionados a Análise de Sentimentos baseada em aspectos e em documento. Após, são apresentados os materiais e métodos utilizados no desenvolvimento desse trabalho, em seguida, os resultados são apresentados e discutidos. Por fim, a conclusão aborda as possibilidades de trabalhos futuros.

\section{Análise de Sentimentos}

A opinião de indivíduos sobre algo sempre foi uma informação importante no processo de tomada de decisões (PANG; LEE, 2008). Esse comportamento tem tomado proporções até pouco tempo não previstas, visto que opiniões e ideias são facilmente compartilhadas por milhões de pessoas através da internet. Essas opiniões simbolizam uma grande quantidade de dados gerados diariamente.

Esses dados são valiosos para organizações e empresas, porém representam um problema devido ao seu volume. Nesse contexto, técnicas da computação podem ser aplicadas em conjunto com métodos e conceitos da área da análise de sentimento ou Mineração de opiniões. Análise de sentimentos, também conhecida como mineração de opinião, é um campo de estudo que analisa opiniões, sentimentos e avaliações de pessoas direcionados a entidades como produtos, serviços e organizações (LIU, 2012). Essa é uma das áreas de pesquisa mais ativas em processamento de linguagem natural e mineração de dados (LIU, 2017). A análise de sentimentos pode ser dividida em três níveis de detalhamento que são: Documento, Sentença e Aspectos ou Entidades (LIU, 2012). Cada tipo de análise representa uma forma diferente de extração de conhecimento, ou seja, cada nível representará um nível diferente da opinião do autor em um corpo de texto, comumente chamado de corpus.

A análise de sentimentos realizada no nível de aspecto ou entidade tem o objetivo de classificar cada opinião do autor sobre cada aspecto de uma ou mais entidades avaliadas e representa o maior nível de detalhamento possível para tarefas do campo de análise de sentimentos. "Em vez de olhar para construções de linguagem (documentos, parágrafos, sentenças, cláusulas ou frases), o nível de aspecto olha diretamente para a própria opinião." (LIU, 2012, pág. 11). Esse grau é considerado o mais complexo, visto que a identificação e classificação de aspectos e suas respectivas opiniões e entidades envolvem um conjunto de desafios da área de processamento de linguagem natural (PLN), por exemplo, identificar aspectos implícitos, entender o contexto de uma palavra em uma frase. De forma sucinta, uma opinião pode ter polaridades diferentes dependendo do contexto, um exemplo disso pode ser visto na sentença "A água da praia estava gelada demais". Nesse exemplo o autor expressa uma opinião negativa sobre a temperatura da água, a polaridade desse tipo de opinião é de difícil classificação visto que gelado pode ter polaridade positiva ou negativa dependendo do contexto. Segundo Liu (2012) esse grau de detalhamento da informação é importante, pois permite vários tipos de análises qualitativas e quantitativas.

O nível de sentença é considerado um passo intermediário entre Aspecto e Documento e avalia as sentenças/frases de um corpus. Um dos desafios desse grau de detalhamento é identificar quais sentenças possuem natureza opinativa sobre a entidade avaliada. Diferenças fundamentais entre a classificação de sentimentos no nível de sentença e de documento são praticamente inexistentes, visto que, sentenças também podem ser avaliadas como documentos menores (LIU, 2012).

A análise de sentimentos também pode ser feita considerando o documento inteiro como uma unidade. Semelhante a análise para o nível de sentença, o nível de documento também requer que seja identificado se o documento avaliado é realmente opinativo. Segundo Cambria et al. (2017), esse tipo de documento contém um conjunto de opiniões sobre um 
conjunto de aspectos de uma ou mais entidades, possui um ou um conjunto de autores e foi feita em um tempo particular. Segundo Liu (2012) esse nível de análise assume que o documento expressa opiniões sobre uma única entidade (Ex. Hotel, Restaurante). As avaliações apresentadas neste trabalho foram feitas a partir de um módulo que realiza análises nesse nível de detalhamento.

\section{Trabalho relacionado - SentimentALL}

O estudo desenvolvido neste artigo foi realizado com base em análises feitas por Oliveira, Marinho e Brito (2018), Araújo (2017) e Brito (2018) utilizando a ferramenta SentimentALL, também desenvolvida por esses dois autores. Segundo Araújo (2017), essa ferramenta tem como objetivo realizar a mineração de opiniões oriundas de sites da internet escritas em Português do Brasil. A Figura 1 representa a arquitetura da SentimentALL.

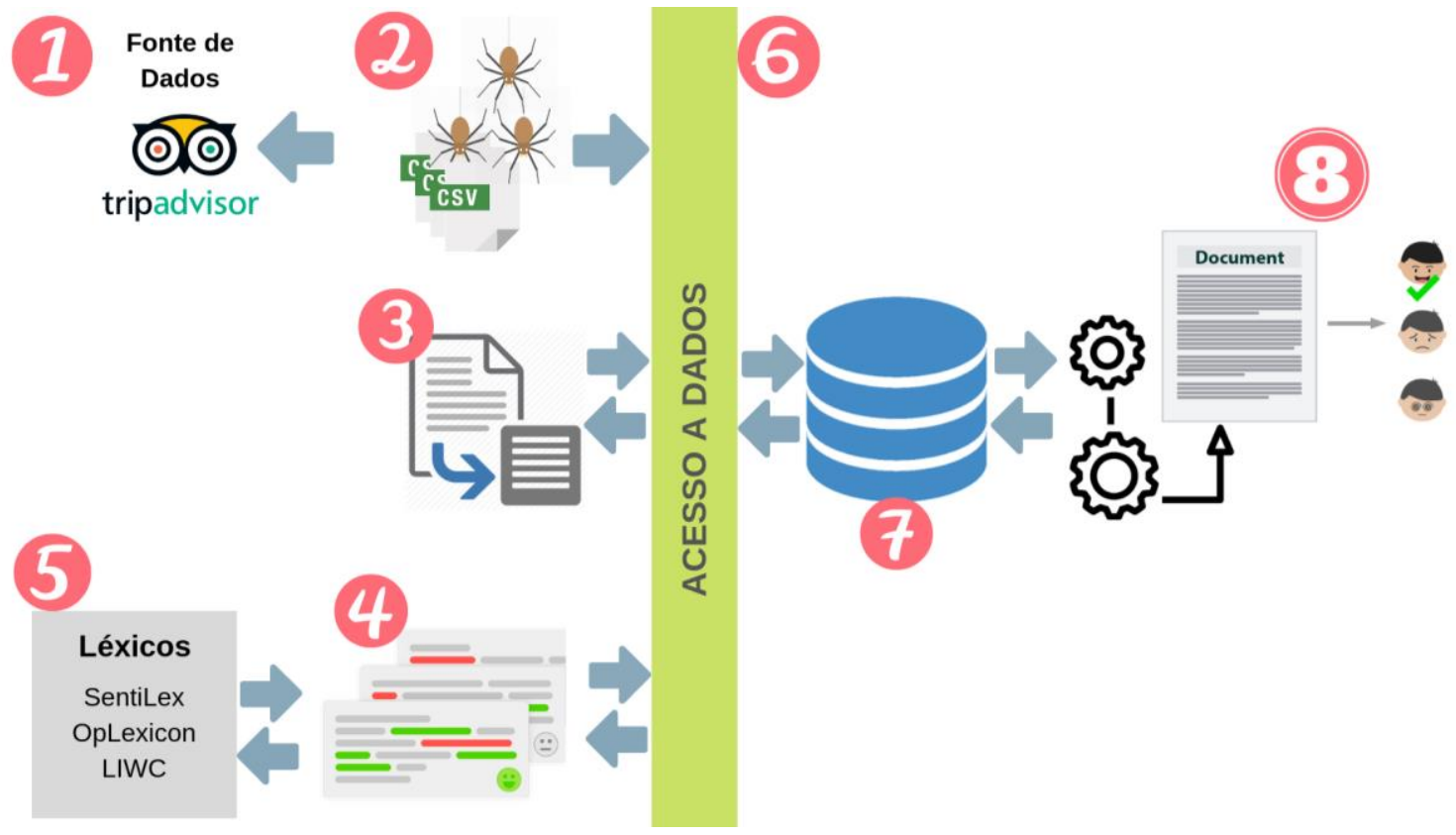

Figura - 1 Arquitetura SentimentALL

A ferramenta SentimentALL desenvolvida por Araújo (2017) e Brito (2018) utiliza como fonte de dados avaliações sobre destinos no Brasil escritas em Português, extraídas do website TripAdvisor (Figura 1-1). O site oferece espaço para que os usuários avaliem acomodações, restaurantes e atrações de uma grande quantidade de destinos no mundo inteiro. Para realizar a coleta desses dados são utilizados Spiders (Figura 1-2). Esses Spiders ou Crawlers são softwares capazes de visitar sistematicamente páginas HTML e extrair dessas páginas dados definidos como importantes para o escopo do projeto. No contexto da ferramenta SentimentALL são coletados, por exemplo, o texto dos comentários/avaliações sobre destinos turísticos e dados complementares como cidade do Autor, data da publicação da avaliação, pontuação dada pelo usuário por meio da escala Likert do site, entre outros. $\mathrm{O}$ resultado dessa etapa são documentos de texto no formato CSV. A Figura 1-3 representa a segunda etapa da ferramenta que faz o pré-processamento dos dados e é composta por um conjunto de técnicas da área de processamento de linguagem natural, essas técnicas são: Normalização de dados, Tokenização, POS Tagging e PMI (Pointwise Mutual Information). Essa etapa tem como resultado dados estruturados que possuem informações suficientes para que sejam usados na etapa de análise.

A Figura 1-4 representa a estrutura para o processo de análise de sentimento no nível de aspecto, nessa etapa os comentários que foram processados anteriormente passam por um algoritmo de classificação de Dependências sintáticas. "a noção fundamental de dependências 
é baseado na ideia de que a estrutura sintática de uma frase consiste de relações binárias assimétricas entre palavras da frase" (NIVRE, 2005, pág. 3, tradução nossa). De forma sucinta, essas dependências definem o relacionamento entre entidades numa frase. Essa estrutura é importante para o processo de identificação da opinião do autor sobre determinado aspecto. Após identificar aspectos e opiniões relacionadas é feita classificação da polaridade dessas opiniões. A classificação de polaridade é feita com base em léxicos de adjetivos representados na Figura 1-6. Os resultados das etapas do processo de análise de sentimentos no nível de aspecto são enviados para um Banco de Dados (Figura 1-7) através da Camada de Acesso que são funções que auxiliam na obtenção e carga de dados (Figura 1-6). A Figura 1-8 representa o módulo de análise no nível de documento apresentado por Oliveira, Marinho e Brito (2018). Esse módulo utiliza cálculos estatísticos que são usados para definir o peso dos aspectos identificados e classificados na SentimentALL e, a partir disso, realizar a atribuição da polaridade geral do comentário. Este trabalho avalia os resultados obtidos a partir desse módulo (Figura 1-8).

\section{Metodologia}

Esta pesquisa é de natureza qualitativa, pois consiste na análise de interpretação dos comentários polarizados por meio do uso do sistema em relação aos resultados da avaliação manual. A amostra utilizada para a avaliação do desempenho é resultado do processo de extração, normalização e classificação de informações realizados por Araújo (2017) e Brito (2018) no trabalho de desenvolvimento da ferramenta SentimentALL versão 2 e dos resultados obtidos no módulo de análise da polaridade geral dos comentários desenvolvido em Oliveira, Marinho e Brito (2018). Nos dois primeiros trabalhos foram analisados um total de 4.597.242 de comentários, das mais de 6.000.00 avaliações públicas extraídas do web site de turismo TripAdvisor ${ }^{\circledR}$. Todos os comentários estão escritos em língua portuguesa e estão relacionados a pontos turísticos, hotéis e restaurantes de destinos turísticos brasileiros. No último trabalho, foi analisada a polaridade geral de 100 comentários.

Para a avaliação do módulo de identificação da polaridade geral dos comentários, foram realizados os passos a seguir:

- Seleção dos comentários: a partir da amostra inicial, foram selecionados 70 comentários de forma aleatória, para que sob os mesmo fosse realizado p processo de análise;

- Organização dos comentários selecionados: os comentários selecionados foram organizados em planilhas a fim de facilitar a análise dos dados;

- Análise manual: consiste na criação de um conjunto de controle, onde a partir dos comentários selecionados foi aplicado o processo de análise de sentimentos - nível documento - de forma manual;

- Criação da matriz de confusão para informações: Para o conjunto de resultados (análise manual - análise equivalente do sistema), foi criada uma matriz de confusão, cujo objetivo é a identificação quantitativa dos comentários positivos que foram classificados como positivos (Verdadeiros-Positivos ou $V_{P}$ ), dos comentários negativos que foram classificados como positivo (Falsos-Positivos ou $F_{P}$ ), dos comentários negativos que foram classificados como negativos (Falsos-Negativos ou $F_{N}$ ) e dos comentários positivos que foram classificados como negativos (VerdadeirosNegativos ou $V_{N}$ ). Um exemplo da matriz de confusão é apresentado na Tabela 1. 


\section{Tabela 1. Matriz de confusão}

\begin{tabular}{|c|c|c|}
\hline$\#$ & Positivo & Negativo \\
\hline Verdadeiro & $\mathrm{V}_{\mathrm{P}}$ & $\mathrm{V}_{\mathrm{N}}$ \\
\hline Falso & $\mathrm{F}_{\mathrm{P}}$ & $\mathrm{F}_{\mathrm{N}}$ \\
\hline
\end{tabular}

- Aplicação das métricas "Precision" e "Recall" para análise de desempenho: Utilizando as informações das matrizes de confusão, foram aplicadas as fórmulas de "Precision" e "Recall" para indicar o índice de desempenho do sistema em relação a identificação da polaridade do comentário;

Análise de correlação: A correlação realizada foi a de Pearson. Essa correlação gera o coeficiente de correlação de Pearson que mede o grau de correlação linear entre duas variáveis quantitativas. É um índice adimensional com valores situados entre $-1,0$ e 1, que reflete a intensidade de uma relação linear entre dois conjuntos de dados (LIRA, 2004). Para essa análise de correlação foi utilizada a informação da avaliação do usuário via escala Likert do TripAdvisor ${ }^{\circledR}$ e o valor da polaridade geral do comentário que estão presentes na base de dados da SentimentALL.

\section{Resultados}

O processo iniciou com a seleção de 70 avaliações na base de dados da SentimentALL. Essas avaliações foram selecionadas de forma aleatória por um script, e em seguida as informações referente ao ID da avaliação, o comentário e o valor na escala Likert foram armazenados em um arquivo no formato CSV, para mais tarde serem utilizadas na análise manual e de correlação.

A partir do arquivo contendo as avaliações selecionadas, foi executada a análise manual dos comentários para criar um conjunto de controle. Neste conjunto, o comentário de cada avaliação foi polarizado como positivo (recebendo o valor 1), negativo (recebendo o valor -1) ou neutro caso não fosse possível inferir uma polaridade positiva ou negativa ao comentário a partir do seu texto (recebendo o valor 0 ).

Em seguida utilizando a informação do ID das avaliações do arquivo csv, estas foram selecionadas no banco de dados para a realização do processo de identificação da polaridade geral do comentário pelo sistema. A informação da polaridade do comentário da avaliação inferida pelo sistema foi agregada à planilha que já continha os dados da análise manual.

Para a análise de correlação, os valores expressos na escala Likert foram compreendidos de uma forma diferente. Para isso, foi considerado o seguinte critério: 1 e 2 (na escala Likert) $=-1$ (para a análise de correlação), 3 (na escala Likert) foi desconsiderado para a análise de correlação, 4 e 5 (na escala Likert) $=1$ (para a análise de correlação). Desta forma, o valor da escala Likert de cada avaliação foi atualizado com o valor utilizado pelo módulo de identificação da polaridade geral do comentário da SentimentALL, que trabalha com 1 (para positivo) e -1 (para negativo).

As avaliações compreendidas como neutra (valor 0 ) na análise manual ou do sistema foram desconsideradas. Além disso, as avaliações que continham a nota 3 expressa na escala Likert também foram desconsideradas, tendo em vista que essa é a nota que está situada no meio da escala (1 - 5), assim sendo, não foi possível classificá-la inteiramente como positiva ou negativa. Com esse processo, após desconsiderar as avaliações compreendidas como neutras, restou o um total de 50 comentários.

Uma vez que os dados foram devidamente organizados, iniciou-se a próxima etapa, que consistiu na criação de uma matriz de confusão que compara o resultado da análise manual com o resultado obtido pelo sistema. Logo, utilizando o modelo de matriz apresentado 
anteriormente (Tabela 1), uma matriz equivalente foi utilizada na comparação dos resultado (Tabela 2).

Tabela 2. Matriz de confusão das avaliações

\begin{tabular}{c|c|c|c|c|}
\hline & A & B & $c$ & 0 \\
\hline 1 & & & Análise do modelo Estatístico & \\
\hline 2 & & & Positivo & Negativo \\
\hline 3 & Análise Manual & Verdadeiro & 39 & 7 \\
\hline 4 & Análise Manual & Falso & 2 & 2 \\
\hline
\end{tabular}

Os campos presentes na matriz de confusão são referentes a análise manual e a análise realizada pelo sistema. A análise manual e a análise no sistema identificaram de forma equivalente a polaridade positiva de um total de 39 comentários e a polaridade negativa em 7 comentários. Já o total de comentários em que o sistema classificou com a polaridade negativa e a análise manual classificou como positiva foram 2. Os comentários entendidos com a polaridade negativa na análise manual e com polaridade positiva na análise do sistema totalizaram 2 comentários.

Com base nestes resultados, foram aplicadas as métricas Precision e Recall, conforme detalhamento a seguir:

$$
\text { A métrica Precision é definida por precision }=\frac{\text { true positive }}{\text { true positive }+ \text { false positive }} \text {, portanto, }
$$
substituindo pelos dados da matriz de confusão, obtêm-se precision $=\frac{39}{39+2}$, cujo resultado é 0,951. Precision fornece informação sobre Falsos - Positivos, então trata-se de quão bom o modelo é para identificar o resultado de maneira precisa.

A métrica Recall é definida por recall $=\frac{\text { true positive }}{\text { true positive }+ \text { false negative }}$, portanto, substituindo pelos dados da matriz de confusão, obtêm-se recall $=\frac{39}{39+2}$, cujo resultado é 0,951 . Recall indica a relação entre as polaridades positivas corretamente identificadas pelo sistema (considerando a avaliação manual) e todas as previsões que realmente são positivas (ou seja, o conjunto True Positive + False Negative).

Para mensurar o avaliação geral do módulo foi utilizado a métrica F-Measure, definida por $F-$ Measure $=2 * \frac{\text { precision } * \text { recall }}{\text { precisioin }+ \text { recall }}$, que utiliza a média das taxas de Precision e Recall encontradas, portanto, substituindo pelos valores de precision e recall, temseF-Measure $=2 * \frac{0,951 * 0,951}{0,951+0,951}$, cujo resultado é 0,951. Essa é a média harmônica entre precision e recall, e seu valor máximo é 1 . Com F-Measure, quanto mais próximo do valor 1 , melhor a avaliação do módulo.

Para verificar qual a relação existente entre o resultado obtido no módulo e com os dados referentes a avaliação via escala Likert realizada pelos usuários do TripAdvisor ${ }^{\circledR}$, foi realizado a análise de correlação de Pearson. 


\begin{tabular}{|c|c|c|c|}
\hline 4 & A & B & C \\
\hline 1 & Id Avaliação & Polaridade & Escala Likert \\
\hline 2 & r100000545 & 1 & 5 \\
\hline 3 & r100013115 & -1 & 3 \\
\hline 4 & r100016264 & -1 & 5 \\
\hline 5 & $r 100030422$ & 1 & 5 \\
\hline 6 & $r 100043225$ & 1 & 4 \\
\hline 7 & $r 100051235$ & 1 & 4 \\
\hline 8 & $r 100051709$ & 1 & 2 \\
\hline 9 & $r 100078074$ & 1 & 5 \\
\hline
\end{tabular}

Figura 4. Amostrados dados para análise de correlação

A Figura 4 apresenta uma amostra dos dados utilizados para a análise de correlação. Id Avaliação é o identificador da avaliação na base de dados da SentimentALL, a polaridade é o resultado alcançado com o módulo de inferência da polaridade geral do comentário, o valor na Escala Likert é o expressado pelo usuário recuperado da base de dados da SentimentALL.

Tabela 3. Dados para análise de correlação

\begin{tabular}{|c|c|c|c|c|c|c|c|}
\hline \#. & $\begin{array}{c}\text { ID } \\
\text { AVALIAÇÃO }\end{array}$ & POLARIDADE & $\begin{array}{l}\text { ESCALA } \\
\text { LIKERT }\end{array}$ & \#. & ID AVALIAÇÃO & POLARIDADE & $\begin{array}{l}\text { ESCALA } \\
\text { LIKERT }\end{array}$ \\
\hline 1 & r100000545 & 1 & 1 & 26 & r100210361 & 1 & 1 \\
\hline 2 & r100016264 & -1 & 1 & 27 & r100212285 & 1 & 1 \\
\hline 3 & r100030422 & 1 & 1 & 28 & r100216188 & 1 & 1 \\
\hline 4 & r100043225 & 1 & 1 & 29 & r100216692 & 1 & 1 \\
\hline 5 & r100051235 & 1 & 1 & 30 & r100217410 & 1 & 1 \\
\hline 6 & r100051709 & 1 & -1 & 31 & r100261730 & 1 & 1 \\
\hline 7 & r100078074 & 1 & 1 & 32 & r100262747 & -1 & -1 \\
\hline 8 & r100080466 & -1 & -1 & 33 & r100266945 & 1 & 1 \\
\hline 9 & r100083167 & -1 & -1 & 34 & r100272442 & -1 & -1 \\
\hline 10 & r100108630 & 1 & 1 & 35 & r100272466 & 1 & 1 \\
\hline 11 & r100120511 & 1 & 1 & 36 & r100277235 & 1 & 1 \\
\hline 12 & r100122191 & 1 & 1 & 37 & r100293130 & 1 & 1 \\
\hline 13 & r100135625 & 1 & 1 & 38 & r100325495 & -1 & -1 \\
\hline 14 & r100157458 & 1 & 1 & 39 & r100365140 & 1 & 1 \\
\hline 15 & r100160671 & 1 & 1 & 40 & r100371377 & 1 & 1 \\
\hline 16 & r100165846 & 1 & 1 & 41 & r100371856 & -1 & 1 \\
\hline 17 & r100170847 & 1 & -1 & 42 & r100397989 & 1 & 1 \\
\hline 18 & r100178645 & 1 & 1 & 43 & r100410747 & -1 & -1 \\
\hline
\end{tabular}




\begin{tabular}{c|c|c|c|c|c|c|c}
19 & $\mathrm{r} 100161573$ & 1 & 1 & 44 & $\mathrm{r} 100431058$ & 1 & 1 \\
\hline 20 & $\mathrm{r} 100182253$ & 1 & 1 & 45 & $\mathrm{r} 100432458$ & 1 & 1 \\
\hline 21 & $\mathrm{r} 100197410$ & 1 & 1 & 46 & $\mathrm{r} 100434833$ & 1 & 1 \\
\hline 22 & $\mathrm{r} 100200658$ & 1 & 1 & 47 & $\mathrm{r} 100445288$ & 1 & 1 \\
\hline 23 & $\mathrm{r} 100207271$ & 1 & 1 & 48 & $\mathrm{r} 100480278$ & 1 & 1 \\
\hline 24 & $\mathrm{r} 100208063$ & 1 & 1 & 49 & $\mathrm{r} 100488011$ & -1 & -1 \\
\hline 25 & $\mathrm{r} 100209906$ & 1 & 1 & 50 & $\mathrm{r} 100493981$ & 1 & 1
\end{tabular}

A Tabela 3 apresenta os dados preparados para a análise de correlação. A coluna polaridade contém o resultado da inferência da polaridade feita pelo sistema. Os valores da escala Likert estão expressos de forma equivalente ao resultado obtido pelo sistema, conforme explicação anterior. O resultado obtido após a análise de correlação de Pearson foi o coeficiente de correlação igual a 0,7289 .

O coeficiente de correlação pode ser interpretado da seguinte forma (LIRA, 2004): 0.9 para mais ou para menos indica uma correlação muito forte; 0.7 a 0.9 positivo ou negativo indica uma correlação forte; 0.5 a 0.7 positivo ou negativo indica uma correlação moderada; 0.3 a 0.5 positivo ou negativo indica uma correlação fraca; 0 a 0.3 positivo ou negativo indica uma correlação desprezível. Portanto, o resultado obtido na análise de correlação entre o resultado do módulo de inferência da polaridade geral do comentário e os dados da avaliação via escala Likert do usuário na avaliação do TripAdvisor ${ }^{\circledR}$ sendo 0,7289 , apresenta uma correlação positiva forte.

\section{Considerações Finais}

Para o desenvolvimento do presente trabalho, foi analisado o indicador de qualidade Conceito Enade, disponível no portal do INEP, órgão responsável pela publicação dos dados. Os indicadores de qualidade são expressos em escala contínua e em cinco níveis, sendo que os níveis iguais ou superiores a 3 (três) indicam qualidade satisfatória. As etapas do trabalho foram definidas conforme apresentado na Figura 1.

A utilização das métricas Precision, Recall e F-Measure mostrou o quão efetivo é o módulo de identificação de polaridade geral para inferir corretamente o status positivo ou negativo a um comentário. Nesse sentido, a correlação corroborou com o entendimento de que o módulo está apresentando resultados, de fato, significativos. Isso porque considerou que o valor na escala Likert dada pelo usuário em uma determinada avaliação possui uma correlação direta com o seu texto em forma de comentário na mesma avaliação. E o valor encontrado mostrou que essa correlação, de fato, existe de forma consistente.

Após os resultados obtidos neste trabalho, é possível levantar, também, alguns questionamentos relacionados a como melhorar o desempenho do módulo, por exemplo, na atribuição dos pesos dos aspectos ou na inferência da polaridade geral. Zhang et al (2011) apresentam em seu trabalho o processo utilizado para realizar a análise de sentimentos no nível do documento na língua chinesa. Em seu processo, Zhang et al (2011) apresentam os critérios utilizados para a definição do peso de cada sentença. Os critérios que o autor coloca são:

- posição da sentença (wp): dado um documento $\mathrm{D}=\{\mathrm{s} 1, \mathrm{~s} 2, \ldots, \mathrm{sN}\}$ sendo um conjunto de frases, onde si é a sentença, a posição de uma sentença em um documento pode indicar sua importância. As sentenças iniciais e finais são frequentemente sentenças temáticas e por isso são consideradas mais importantes que às demais. 
Assim sendo, são atribuídos pesos mais altos às sentenças nas duas extremidades do documento. O recurso de posição (wp) de uma frase é definido como:

onde $1 \leq \mathrm{i} \leq \mathrm{n}$.

$$
w p(s i)=\frac{1}{\min (i, N-i+1)}
$$

- os termos com pesos na sentença (wt): referem-se às sentenças que contêm os termos mais importantes. A ocorrência de termos em uma sentença pode indicar sua significância em um documento. Ou seja, a importância aumenta proporcionalmente ao número de vezes que uma palavra aparece na frase e no documento.

- semelhança entre a sentença e o título (wh): trata da similaridade entre uma frase e o título. Já que o título pode ser considerado um resumo do documento, logo, uma sentença (w) semelhante para ao título (h) deve contribuir mais para o documento.

- ocorrência de palavras-chave (wk): mede o número total de palavras-chave que ocorrem na frase. A frequência de palavras-chave na frase também indica a relevância e importância da sentença para o documento. Zhang et al (2011) utilizam a abordagem de extração de palavras-chave de Matsuo e Ishizuka (2004) para identificar palavraschave no documento.

- modo de primeira pessoa (wf): indica se a sentença está em o modo de primeira pessoa. Zhang et al (2011) afirmam que frases em modo de primeira pessoa, indicadas por pronomes como eu/e/nós, tendem a ter mesma polaridade do documento inteiro.

Dessa forma, com base no modelo de Zhang et al (2011) é possível que, em trabalhos futuros, o modelo estatístico no qual é utilizado no módulo de inferência da polaridade geral dos comentários do TripAdvisor ${ }^{\circledR}$ analisados na SentimentALL seja incrementado com novas regras com o intuito de melhorar o resultado obtido, além do estudo de novas formas para a análise do desempenho do módulo.

\section{Referências}

ARAÚJO, Luan Gomes de Almeida. SENTIMENTALL VERSÃO 2: Desenvolvimento de Análise de Sentimentos em Python. 2019. 123 f. TCC (Graduação) - Curso de Ciência da Computação, Centro Universitário Luterano de Palmas, Palmas, 2017.

BAKER, A. (2008). Computer Aided Invariant Feature Selection. (Doctoral Dissertation). University of Florida. Retrieved from. Disponível em: <http://ufdc.ufl.edu/UFE0022870>. Acesso em: 20 de abril de 2019.

BRITO, Parcilene Fernandes de. RELATOS VERBAIS DE CONSUMIDORES EM AVALIAÇÕES ON-LINE: PROSPECÇÃO COMPUTACIONAL E INTERPRETAÇÕES COM BASE NO BEHAVIORAL PERSPECTIVE MODEL (BPM). $2018.182 \mathrm{fl}$. Tese (Programa de Pós-Graduação STRICTO SENSU em Psicologia) - Pontifícia Universidade Católica de Goiás, Goiânia-GO.

CAMBRIA, Erik; DAS, Dipankar; BANDYOPADHYAY, Sivaji; FERACO, Antonio. Affective Computing and Sentiment Analysis. In: CAMBRIA, Erik; DAS, Dipankar; BANDYOPADHYAY, Sivaji; FERACO, Antonio (Ed.). A Practical Guide to Sentiment Analysis. 5. ed. [s.1.]: Springer, 2017. Cap. 1. p. 1-10. (Socio-Affective Computing). Disponível em: <http://ww.sentic.net/practical-guide-to-sentiment-analysis.pdf $>$. Acesso em: 14 abr. 2019.

DOMINGUES, Miriam Lúcia Campos Serra. ABORDAGEM PARA O DESENVOLVIMENTO DE UM ETIQUETADOR DE ALTA ACURÁCIA PARA O 
PORTUGUÊS DO BRASIL. 2011. 137 f. Tese (Doutorado) - Curso de Programa de Pósgraduação em Engenharia Elétrica, Instituto Tecnológico, Universidade Federal do Pará, Belém, 2011.

LIRA, Sachiko Araki. ANÁLISE DE CORRELAÇÃO: ABORDAGEM TEÓRICA E DE CONSTRUÇÃO DOS COEFICIENTES COM APLICAÇÕES. 2004. 196 f. Dissertação apresentada ao Curso de Pós Graduação. Universidade Federal do Paraná. Disponível em: $<$ http://www.ipardes.gov.br/biblioteca/docs/dissertacao_sachiko.pdf $>$. Acesso em: Acesso em: 20 abr. 2019.

LIU, Bing. Many Facets of Sentiment Analysis. In: CAMBRIA, Erik; DAS, Dipankar; BANDYOPADHYAY, Sivaji; FERACO, Antonio (Ed.). A Practical Guide to Sentiment Analysis. 5. ed. Illinois: Springer, 2017. Cap. 2. p. 11-39. Disponível em: $<$ http://ww.sentic.net/practical-guide-to-sentiment-analysis.pdf $>$. Acesso em: 20 abr. 2019.

LIU, Bing. Sentiment Analysis and Opinion Mining. [s.l.]: Morgan \& Claypool Publishers, 2012. $168 \mathrm{p}$.

MATSUO, Y., \& ISHIZUKA, M. (2004). Keyword extraction from a single document using word co-occurrence statistical information. International Journal on Artificial Intelligence Tools, 13(1), 157-169.

NIVRE, Joakim. Dependency Grammar and Dependency Parsing. 2005. Disponível em: $<$ http://stp.lingfil.uu.se/ nivre/docs/05133.pdf>. Acesso em: 21 abr. 2019.

OLIVEIRA, Taylor Santos; MARINHO, Dionnys Santos; BRITO, Parcilene Fernandes de. Módulo de identificação da polaridade geral dos Comentários do TripAdvisor baseado na polaridade dos seus Aspectos. 2018. 11 f. ENCOINFO - 2018. PANG B. e LEE L., Opinion Mining and Sentiment Analysis, Foundations and Trends $\mathrm{R}$ in Information Retrieval, vol 2, nos 1-2, pp 1-135, 2008.

PANG, Bo et al. Opinion mining and sentiment analysis. Foundations and Trends ${ }^{\circledR}$ in Information Retrieval, v. 2, n. 1-2, p. 1-135, 2008.

RAMOS, Nelson Azoubel. Avaliação e Comparação de Desempenho de Computadores: Metodologia e Estudo de Caso. 2008. 60 f. Universidade Federal de Pernambuco: Centro de Informática Curso de Graduação em Engenharia da Computação. Disponível em: $<$ http://www.cin.ufpe.br/ tg/2008-2/nar.pdf>. Acesso em: 20 de abril de 2019.

ZHANG, Changli et al. Sentiment Analysis of Chinese Documents: From Sentence to Document Level. 2011. 15 f. Journal of the American Society for Information Science and Technology. Disponível em: <https://www.researchgate.net/publication/220433795Ver>. Acesso em: 20 de abril de 2019. 